\section{Fluorose dentária:}

\section{comparação de dois estudos de prevalência}

\author{
Dental fluorosis: \\ comparison of two prevalence studies
}

Paulo Frazão 1

Adriana Campos Peverari 2

Tania Izabel Bighetti Forni 2

Anderson Gomes Mota 2

Leslie Roberta da Costa 3

\author{
1 Programa de Pós-graduação \\ em Saúde Coletiva, \\ Universidade Católica \\ de Santos, Santos, Brasil. \\ 2 Faculdade de Odontologia, \\ Universidade Metodista \\ de São Paulo, São Bernardo \\ do Campo, Brasil. \\ 3 Secretaria da Saúde, \\ Prefeitura Municipal \\ de Ribeirão Pires \\ Ribeirão Pires, Brasil. \\ Correspondência \\ P. Frazão \\ Programa de Pós-graduação \\ em Saúde Coletiva \\ Universidade \\ Católica de Santos. \\ Rua Carvalho de Mendonça \\ 144, 4 o andar, Santos, SP \\ 11070-906, Brasil. \\ pafrazao@usp.br
}

\section{Abstract}

Dental fluorosis is an enamel opacity resulting from the ingestion of excessive amounts of fluoride (chronic exposure) during tooth formation. Estimation of this problem among populations exposed to fluoride sources is necessary and should be performed periodically to monitor changes in levels and patterns in the disorder. This research aimed to describe and compare two prevalence studies to estimate the occurrence of dental fluorosis in a population of schoolchildren in Ribeirão Pires, São Paulo State, Brazil, conducted in 1997 and 2000. The methods used in each study are described briefly and the results compared. Methodological aspects and implications for public health are discussed.

Public Health Dentistry; Dental Fluorosis; Prevalence Studies

\section{Introdução}

Fluorose dentária é um distúrbio de desenvolvimento que afeta o esmalte durante sua formação, sendo provocada pela ingestão excessiva e prolongada de flúor. Ocorre por depósitos de flúor na estrutura dentária, levando a alterações nos ameloblastos, modificações na homeostase do cálcio e na formação dos cristais de apatita 1. Evidências indicam que os ameloblastos são mais afetados durante a maturação pré-eruptiva 2,3 . As manifestações vão depender da quantidade de flúor ingerida, do tempo de exposição, da idade, do peso e estado nutricional do indivíduo.

O declínio da cárie e o aumento da prevalência da fluorose nas formas mais leves têm sido observados em diferentes regiões do mundo. Alguns autores notaram maior aumento proporcional em locais com teores deficientes de flúor na água 4,5; outros observaram diferenças pequenas entre áreas fluoretadas e não fluoretadas 6,7; e outros, sem entrar no mérito do seu aumento, atribuíram a ocorrência das formas leves à fluoretação da água 8 .

Em nosso país, a prevalência de fluorose dentária tem sido avaliada mais regularmente nos últimos anos, período no qual a exposição da população infantil a diferentes métodos de uso do flúor tem aumentado. Levantamentos mostrando o declínio na cárie dentária têm revelado que a fluorose começa a surgir. Em revi- 
são crítica, Cangussu et al. 9 discutem esse distúrbio como um relevante problema de Saúde Coletiva. Para os autores, embora com poucos casos de severidade, os resultados sugerem freqüências mais altas do que as esperadas, sendo necessárias medidas de vigilância à saúde bucal.

Para Cury \& Tabchoury 10, há uma divisão de opiniões entre os profissionais, e, embora a maioria considere que a prevalência não é um problema de Saúde Coletiva, em concordância com a percepção da população, as crianças têm sido expostas a fontes múltiplas de flúor, que, consideradas em conjunto, significam ampla variação na dose adequada.

Há unanimidade, entretanto, em reconhecer a necessidade de maior precisão e fortalecimento das medidas de vigilância à saúde, incluindo ações no campo da epidemiologia, da vigilância sanitária, da prescrição e uso de produtos fluorados e da educação em saúde bucal.

Em relação à epidemiologia, Forni 11 indicou a necessidade de investigações para comparar os critérios de determinação dos dentes

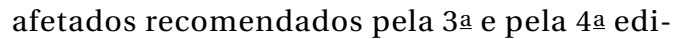
ções do manual Oral Health Surveys da Organização Mundial da Saúde (OMS) 12,13. Investigando a literatura, Moysés et al. 14 identificaram problemas relativos à confiabilidade dos dados disponíveis sobre fluorose dentária. O objetivo desta pesquisa foi comparar dois estudos de prevalência conforme os procedimentos metodológicos empregados e as estimativas obtidas, discutindo as implicações para a Saúde Coletiva.

\section{Métodos}

\section{Fonte dos dados}

O Município de Ribeirão Pires, criado em 1953, está situado a sudeste da Região Metropolitana de São Paulo, tendo como limites os municípios de Santo André, Mauá, Rio Grande da Serra e Suzano. A partir de 1976, foi considerado área de proteção de mananciais das bacias hidrográficas dos reservatórios da Billings, Taiaçupeba e Guaió. Após a criação da legislação, foram aprovados projetos de ocupação do solo destinados a residências de veraneio. Sua vegetação constituinte é de floresta tropical, notadamente a Mata Atlântica, onde predomina o clima tropical de altitude, com temperatura máxima de $32^{\circ} \mathrm{C}$, mínima de $5^{\circ} \mathrm{C}$ e média de $16^{\circ} \mathrm{C}$. Segundo dados relativos a 1999 da Fundação Instituto Brasileiro de Geografia e Estatística, a população estimada era de 108.121 habitantes. A maior parte da população tinha acesso à água fluoretada desde 1984, sendo que apenas os bairros de Jardim Iramaia, Jardim Santista, Jardim Pereira Barreto, Jardim Lusitano e Chácara Viana não possuíam rede distribuidora de água. Em 1996, teve início um controle sistemático mensal dos teores de flúor na água de abastecimento público do município, através de um programa de vigilância sanitária coordenado pela Direção Regional de Saúde II da Secretaria de Estado da Saúde de São Paulo (DIR-II).

No município, eram desenvolvidas ações coletivas e de assistência odontológica individual, nas quais a urgência odontológica era assegurada a todos os cidadãos, e as ações programáticas eram dirigidas a grupos, social e epidemiologicamente mais vulneráveis, com ênfase à população de pré-escolares e escolares e a grupos sociais específicos que freqüentavam as unidades básicas de saúde. Em 1997, o município dispunha de 42 cirurgiões-dentistas exercendo atividades na rede pública. Nesse mesmo ano, foi realizado um estudo epidemiológico transversal pela Secretaria de Estado da Saúde de São Paulo em parceria com a Faculdade de Saúde Pública da Universidade de São Paulo e apoio da Secretaria Municipal de Saúde de Ribeirão Pires, cujos objetivos foram estimar a prevalência de cárie dentária na população de 5 a 15 anos de idade e a prevalência de fluorose e oclusopatias na amostra do estudo. Aos 12 anos de idade, $34 \%$ dos escolares estavam livres de cárie. $\mathrm{O}$ ataque de cárie nessa idade foi de 2,07 , um valor que caracterizava uma prevalência baixa 15 .

No ano 2000, novo estudo epidemiológico transversal foi efetuado pela Secretaria Municipal da Saúde com o apoio da Universidade Metodista de São Paulo, cujo propósito foi estimar a prevalência de fluorose dentária nos adolescentes do município 16 .

\section{Amostra dos estudos}

Em ambos os estudos, a população de referência correspondeu às crianças em idade escolar residentes no município, sendo que a escola foi definida como unidade amostral por razões operacionais e em virtude de uma proporção bastante significativa da população de referência freqüentar esses espaços. No estudo A, fo- 
ram sorteadas 12 escolas, e, no estudo B, 20 estabelecimentos. A composição dos elementos amostrais também obedeceu a critério aleatório, com a diferença que, no primeiro estudo, foi feito sorteio simples utilizando tabela de números equiprováveis, e, no segundo, sorteio sistemático. Na Tabela 1, são apresentadas as características das amostras.

\section{Instrumento de medida}

Para a observação do esmalte dentário em relação à presença de sinais de fluorose, foi empregado, em ambos os estudos, o índice de $D e$ $a n$, com a diferença que, no estudo A, foi adotada a versão proposta pela OMS em 1987, e, no estudo B, a versão publicada em 1997. Em ambas as versões, são preconizados os seguintes códigos para cada categoria:

- Normal $\{0\}$ : esmalte superficial liso, brilhante e geralmente de cor branca bege pálida;

- Questionável $\{1\}$ : o esmalte apresenta leves aberrações na translucidez de esmalte normal, que podem variar desde pequenos traços esbranquiçados até manchas ocasionais;

- Muito leve \{2\}: áreas pequenas e opacas de cor branca porosas dispersas irregularmente sobre o dente, mas envolvendo menos de $25 \%$ da superfície dentária vestibular;

- Leve $\{3\}$ : a opacidade branca do esmalte é mais extensa do que para o código 2, mas recobre menos de $50 \%$ da superfície dentária;

- Moderado \{4\}: a superfície de esmalte dos dentes apresenta um desgaste acentuado e manchas marrons freqüentemente alterando a anatomia do dente;

- Severo $\{5\}$ : a superfície do esmalte está muito afetada, e a hipoplasia é tão acentuada que o formato geral do dente pode ser afetado. Existem áreas com fóssulas ou desgastes, e as manchas marrons estão espalhadas por toda parte;

Tabela 1

Características das amostras segundo o estudo. Ribeirão Pires, São Paulo, Brasil, 1997 e 2000.

\begin{tabular}{lcc}
\hline Características & Estudo A & Estudo B \\
\hline Ano de realização & 1997 & 2000 \\
Unidades amostrais & 12 & 20 \\
Grupo etário (anos) & $5-15$ & 12 \\
Tamanho da amostra & 733 & 307 \\
Taxa de não resposta & 4,8 & 20,9
\end{tabular}

Fontes: Faculdade de Saúde Pública, Universidade de São Paulo/

Secretaria de Estado da Saúde de São Paulo 15; Peverari et al.16. os dentes freqüentemente apresentam uma aparência de corrosão.

A diferença entre as publicações diz respeito ao padrão final para a classificação do indivíduo: enquanto, na versão de 1987, o registro toma por base, sem distinção, os dois dentes mais afetados; na segunda, o registro deve corresponder ao dente menos alterado entre os dois mais afetados, com a finalidade de não superestimar a ocorrência do problema na população.

Em ambos os estudos, nos casos em que, pela condição bucal do indivíduo, não foi possível visualizar claramente os dentes, o elemento amostral foi excluído do exame, sendo registrado o código 8. Nos casos em que o dente se encontrava em erupção, o indivíduo foi classificado se o dente homólogo estivesse presente com pelo menos $50 \%$ de sua coroa visível, caso contrário, esse elemento amostral também foi excluído.

\section{Preparo dos examinadores e aferição do evento}

Os examinadores foram submetidos a treinamento em ambos os estudos. No estudo A, a concordância percentual foi apurada seguindo recomendação da OMS 12, enquanto, no estudo B, foi calculado o coeficiente kappa ${ }^{17}$. Na Tabela 2, são mostradas as características do treinamento em cada estudo e o grau de homogeneidade nas observações alcançado pelos examinadores.

\section{Coleta dos dados}

Em ambos os estudos, os exames foram realizados em ambiente escolar, sempre com a presença de luz natural. No estudo B, o relatório afirma que os exames foram interrompidos na ausência dessas condições. A presença de neblina cobrindo o município em alguns períodos foi razão para essa interrupção. Enquanto, no primeiro estudo, os examinadores coletaram dados sobre outras condições de saúde bucal (oclusão, aspectos da coroa e necessidade de tratamento correspondente a cada dente) utilizando sonda exploradora e espelho bucal, no segundo estudo, a dentição foi examinada visualmente somente para observar a ocorrência de fluorose dentária com auxílio de espátula de madeira. Cabe destacar que, no estudo B, foi registrado o tipo dentário de referência para a classificação. 
Características do treinamento dos examinadores segundo o estudo. Ribeirão Pires, São Paulo, Brasil, 1997 e 2000.

\begin{tabular}{|c|c|c|c|c|}
\hline Características do treinamento & \multicolumn{2}{|r|}{ Estudo A } & \multicolumn{2}{|c|}{ Estudo B } \\
\hline Número de examinadores & \multicolumn{2}{|r|}{4} & \multicolumn{2}{|r|}{2} \\
\hline Envolveu mais de uma condição bucal & \multicolumn{2}{|r|}{ Sim } & \multicolumn{2}{|r|}{ Não } \\
\hline Duração do treinamento (horas) & \multicolumn{2}{|r|}{20} & \multicolumn{2}{|r|}{36} \\
\hline \multirow[t]{2}{*}{ Número total de exames } & \multicolumn{2}{|r|}{127} & \multicolumn{2}{|r|}{403} \\
\hline & $\%$ & Número de exames & Kappa & Número de exames \\
\hline \multicolumn{5}{|l|}{ Consistência intra-examinador } \\
\hline \multirow[t]{2}{*}{ Durante a calibração } & - & - & 0,78 & 118 \\
\hline & & & 0,79 & 118 \\
\hline Durante a coleta dos dados & 96,8 & 16 & - & - \\
\hline Consistência interexaminador & 73,7 & 38 & 0,80 & 68 \\
\hline
\end{tabular}

Fontes: Faculdade de Saúde Pública, Universidade de São Paulo/Secretaria de Estado da Saúde de São Paulo 15; Peverari et al.16.

\section{Comparação e análise dos dados}

Os dados do estudo A correspondentes às idades de 12 a 15 anos não apresentaram diferenças estatisticamente significativas e foram agrupados para permitir comparação e maior precisão das estimativas. Os dados relativos à variável de interesse, expressos em proporções, foram apresentados em tabelas contendo a distribuição simples das freqüências absolutas e relativas. Os critérios adotados para determinar ausência ou presença de fluorose foram os empregados por Dean \& McKay 18, Galagan \& Lamson 19 e Warnakulasuriya et al. 20. Foi calculado o Índice de Fluorose Dentária da Comunidade (CFI) expresso pela divisão da somatória do produto da freqüência absoluta de cada categoria por um determinado peso (normal - 0; questionável - 0,5 ; muito leve - 1 ; leve 2 ; moderada -3 ; severa -4 ) pelo número de indivíduos examinados. Estimativas por ponto e por intervalo foram calculadas adotando-se um nível de confiança de $95 \%$. Para comparar os resultados em cada sexo, foi aplicado o teste do Qui-quadrado com correção de Yates.

\section{Resultados}

A distribuição da amostra correspondente a cada estudo segundo a condição do esmalte para fluorose dentária é apresentada na Tabela 3.

Conforme pode ser observado, a comparação das estimativas mostrou nítida discrepância. No estudo A, dos escolares examinados
(259), 62,50\% (162) apresentaram esmalte com fluorose dentária, enquanto, no estudo B, dos 307 escolares, 102 mostravam presença de sinais de fluorose dentária correspondendo a $33,22 \%$ da amostra. Dos adolescentes com fluorose, a maioria tanto no estudo A (83/102) quanto no estudo B (153/162) apresentou manifestações incluídas na categoria muito leve. As estimativas de fluorose nas categorias leve e moderada/severa não apresentaram diferenças estatisticamente significativas. Também não foram observadas diferenças segundo o sexo em ambos os estudos.

No estudo realizado em 1997, o CFI proposto por Dean correspondeu a 0,82 - enquanto, em 2000, o cálculo do CFI resultou num valor igual a 0,67 .

Na Tabela 4, são apresentados os dados relativos ao elemento dental de referência para a classificação da fluorose dentária no indivíduo pertencente à amostra do estudo B. Pode-se verificar que o grupo dentário dos pré-molares foi o mais afetado, correspondendo a cerca de $40,0 \%$ dos dentes no maxilar superior e a $20,0 \%$ na mandíbula. O segundo grupo dentário mais afetado correspondeu aos molares numa taxa de $14,6 \%$ no maxilar superior e a $15,7 \%$ na mandíbula. Os incisivos superiores, grupo dentário esteticamente mais importante, foram atingidos em 3,9\% dos casos.

Dos 165 escolares classificados na categoria questionável, dois deles o foram por sinais em caninos, e o restante, em dentes posteriores sem implicações estéticas. 
Número, porcentagem e intervalos de confiança (95\%) da amostra segundo a condição de esmalte para fluorose dentária. Ribeirão Pires, São Paulo, Brasil, 1997 e 2000.

\begin{tabular}{lrrrrrr}
\hline Condição do esmalte & \multicolumn{3}{c}{ Estudo A } \\
& $\mathrm{n}$ & $\%$ & IC95\% & \multicolumn{2}{c}{$\begin{array}{c}\text { Estudo B } \\
\%\end{array}$} & IC95\% \\
\hline Sem fluorose & 97 & 37,5 & $31,5-43,7$ & 205 & 66,8 & $61,2-72,0$ \\
Com fluorose & 162 & 62,5 & $56,3-68,5$ & 102 & 33,2 & $27,9-38,8$ \\
Muito leve & 153 & 59,1 & $52,8-65,1$ & 83 & 27,1 & $22,1-32,4$ \\
Leve & 8 & 3,1 & $1,3-5,9$ & 16 & 5,2 & $3,0-8,3$ \\
Moderada + severa & 1 & 0,4 & - & 3 & 0,9 & $0,2-2,8$ \\
Total & 259 & 100,0 & - & 307 & 100,0 & - \\
\hline
\end{tabular}

Fontes: Faculdade de Saúde Pública, Universidade de São Paulo/Secretaria de Estado da Saúde de São Paulo 15; Peverari et al.16. IC95\%: Intervalo de confiança 95\%.

Tabela 4

Número e porcentagem de elementos dentais segundo a presença de fluorose dentária. Ribeirão Pires, São Paulo, Brasil, 2000.

\begin{tabular}{|c|c|c|c|c|c|c|c|c|}
\hline \multirow[t]{3}{*}{ Elemento dental de referência } & \multicolumn{8}{|c|}{ Graus de fluorose } \\
\hline & \multicolumn{2}{|c|}{ Muito leve } & \multicolumn{2}{|c|}{ Leve } & \multicolumn{2}{|c|}{ Moderada/severa } & \multicolumn{2}{|c|}{ Total } \\
\hline & $\mathrm{n}$ & $\%$ & $n$ & $\%$ & $\mathrm{n}$ & $\%$ & $\mathrm{n}$ & $\%$ \\
\hline \multicolumn{9}{|l|}{ Superiores } \\
\hline Incisivos centrais & 3 & 3,6 & - & - & 1 & 33,3 & 4 & 3,9 \\
\hline Incisivos laterais & - & - & - & - & - & - & - & - \\
\hline Caninos & 1 & 1,2 & - & - & - & - & 1 & 0,9 \\
\hline Primeiro pré-molar & 19 & 22,9 & 3 & 18,7 & - & - & 22 & 21,7 \\
\hline Segundo pré-molar & 16 & 19,3 & 3 & 18,7 & - & - & 19 & 18,7 \\
\hline Primeiro molar & 10 & 12,0 & 2 & 12,6 & 1 & 33,3 & 13 & 12,7 \\
\hline Segundo molar & 2 & 2,5 & - & - & - & - & 2 & 1,9 \\
\hline \multicolumn{9}{|l|}{ Inferiores } \\
\hline Incisivos centrais & - & - & - & - & - & - & - & - \\
\hline Incisivos laterais & - & - & - & - & - & - & - & - \\
\hline Caninos & 2 & 2,5 & - & - & - & - & 2 & 1,9 \\
\hline Primeiro pré-molar & 8 & 9,6 & 3 & 18,7 & - & - & 11 & 10,8 \\
\hline Segundo pré-molar & 10 & 12,0 & 2 & 12,6 & - & - & 12 & 11,8 \\
\hline Primeiro molar & 5 & 6,0 & 3 & 18,7 & 1 & 33,3 & 9 & 8,8 \\
\hline Segundo molar & 7 & 8,4 & - & - & - & - & 7 & 6,9 \\
\hline Total & 83 & 100,0 & 16 & 100,0 & 3 & 100,0 & 102 & 100,0 \\
\hline
\end{tabular}

Fontes: Peverari et al.16. Percentuais com uma casa decimal.

\section{Discussão}

Neste artigo, foram comparados dados de dois estudos epidemiológicos transversais, a partir de amostras probabilísticas, efetuados para estimar a prevalência de fluorose dentária em adolescentes no Município de Ribeirão Pires, uma área onde a população infantil está exposta a fontes múltiplas de flúor. As estimativas obtidas por intervalo de confiança de $95 \%$ apresentaram valores bastante discrepantes que encorajaram os pesquisadores à análise dos procedimentos metodológicos e suas implicações para futuros estudos de prevalência e para a saúde coletiva.

Dada a presença de alguma subjetividade na natureza da classificação, exames de fluoro- 
se dentária podem apresentar mais variação do que aqueles realizados para outras condições de saúde bucal 21. Entretanto, avaliações sobre a reprodutibilidade do índice de Dean têm mostrado excelente concordância, excluída a chance, quando sujeitos foram classificados com respeito à ausência e presença de fluorose. Quando a unidade de análise é o dente, menor concordância tem sido observada 22 .

Em ambos os estudos, os procedimentos necessários para assegurar a casualização da amostra e evitar viés de seleção foram adotados. A taxa de não resposta apresentada pelo estudo B, embora superior ao estudo A, não significou risco para a representatividade da amostra e se manteve em níveis aceitáveis 23 . De modo análogo, embora o estudo A tenha empregado número inferior de unidades amostrais em relação ao estudo B, o valor adotado foi considerado suficiente para permitir a dispersão dos elementos por todas as regiões da cidade.

Uma das hipóteses para a diferença entre as estimativas provavelmente reside no fato de o estudo A seguir a padronização proposta pela $3^{\text {a }}$ edição do manual da OMS, a qual utilizava como referência os dois dentes mais afetados. No estudo B, foi adotada a orientação da $4 \underline{a}$ edição, na qual se classifica o indivíduo tomando como padrão o dente menos acometido entre os dois mais afetados, aspecto importante na medida em que a distinção entre a presença e ausência de fluorose pode depender da inclusão do sujeito no grau questionável ou muito leve das categorias da variável, cuja diferença entre os sinais exige extrema acurácia do observador e pode prejudicar a aferição do evento e comprometer a estimação da prevalência do problema na população.

Além disso, fatores relativos ao processo de calibração e às condições técnicas do exame também devem ser considerados. No estudo B, os examinadores foram orientados a iniciar a coleta dos dados somente após a obtenção de valores consistentes de concordância intra e interexaminadores, o que implicou num processo mais intensivo e com maior duração - 36 horas e 403 exames. Embora de difícil praticidade na medida em que não se dispunha, à exceção da experiência dos pesquisadores, de elementos objetivos para projetar a quantidade de horas necessária, recomenda-se que esse tipo de procedimento seja levado em consideração no planejamento de atividades de calibração em pesquisas para estimar a prevalência da fluorose dentária. Além disso, no estudo A, a avaliação foi efetuada após o exame da característica da coroa em relação à cárie, da necessidade de tratamento dentário e da condi- ção oclusal do indivíduo, enquanto, no estudo B, ela compreendeu o único objeto da pesquisa. Revisando os estudos nacionais e internacionais sobre fluorose dentária no período de 1970-1997, Forni 11 notou maior freqüência no emprego de um a dois examinadores e uma variação de um a oito no número de observadores empregados nas pesquisas. A participação de quatro e dois examinadores, respectivamente em cada estudo, não exerceu influência nos resultados relativos às categorias de maior grau de severidade (leve, moderada e severa).

A despeito dessas diferenças, deve-se observar que, nos dois estudos, o CFI apresentou significado "leve" para Saúde Coletiva alcançando valores de 0,82 em 1997 e 0,67 em 2000. Isso pode ser atribuído ao fato do índice ponderar com maior valor as proporções relativas às categorias moderada e severa. De fato, a ausência de diferença entre essas categorias reduz bastante a probabilidade da existência de significados distintos para a Saúde Coletiva.

Atualmente, e principalmente onde é evidente a necessidade de monitorar com mais precisão a fluorose dentária, a real utilidade desse indicador para essa finalidade tem sido questionada 24. Provavelmente outras medidas irão substituir o CFI para permitir uma avaliação mais precisa dos efeitos biológicos da exposição a fontes múltiplas de flúor 25.

O conhecimento científico sobre o efeito do flúor, especialmente os estudos epidemiológicos clássicos realizados, através do Serviço de Saúde Pública, entre 1933 e 1945, por H. Trendley Dean, em regiões "naturalmente" fluoretadas dos Estados Unidos, demonstrou que o máximo de benefício na prevenção da cárie dentária pelo seu uso é alcançado com a presença de sinais de fluorose muito leve em uma pequena proporção da comunidade. Com base nisso, a presença na população de 10 a $15 \%$ de fluorose muito leve tem sido considerada aceitável. É o ônus a pagar pelo benefício. Na época, foi desenvolvido um indicador para classificar o grau de fluorose na comunidade, cujos valores acima de 0,60 implicavam desde a desfluoretação das águas até o seu abandono.

Associando-se esses conhecimentos ao fato de a água de abastecimento do município ter sido fluoretada a partir de 1984, o que permite supor que todos os adolescentes examinados estiveram expostos a esse benefício, pode-se concluir que existe uma proporção excessiva de fluorose dentária na comunidade, duas vezes superior ao aceitável, e que, portanto, a fluoretação das águas de abastecimento deveria ser interrompida. A facilidade do raciocínio pode levar a conclusões errôneas sem as devidas 
precauções fundamentadas nos conhecimentos científicos.

De fato, a interpretação dos resultados relativos aos estudos de fluorose dentária e suas implicações para a Saúde Coletiva têm desafiado os pesquisadores e os sanitaristas nas últimas duas décadas, especialmente em virtude da multiplicação das fontes de flúor, do risco potencial que isso tem representado para a ocorrência de fluorose e o efetivo aumento observado em sua prevalência em diversas partes do mundo, em regiões com e sem as águas de abastecimento fluoretadas 4,5,7,8,26. Essa elevação tem sido atribuída basicamente à associação de métodos sistêmicos (água + medicamentos + polivitamínicos) e à ingestão de flúor a partir do uso inadequado de produtos tópicos, principalmente cremes dentais na primeira infância.

Na verdade, é preciso distinguir os conhecimentos sanitários produzidos no contexto da fluorose provocada por fonte única relativa à água "naturalmente" fluoretada, contendo teores superiores ao nível ótimo para prevenção da cárie (fluorose dentária endêmica), da fluorose que começa a surgir provocada por exposição a fontes múltiplas de flúor. No estudo B, no qual foram registrados os dentes de referência, a maioria dos sinais indicativos de fluorose foi incluída na categoria muito leve, na qual menos de $25 \%$ da superfície dentária vestibular estava envolvida por pequenas manchas brancas e opacas irregularmente dispersas. Conforme a Tabela 4, os dentes de referência não foram os incisivos superiores, e sim os prémolares e molares. Desses, os pré-molares foram os elementos dentais mais atingidos, fato atribuído ao efeito cumulativo associado ao extenso período de formação do esmalte desses dentes compreendido dos 18 meses aos 7 anos de vida 27,28

Foram observados sinais de fluorose nos graus moderado e severo em $1 \%$ das crianças, $\mathrm{e}$ os dentes posteriores foram os mais afetados. Das 307 crianças, uma apresentou sinais de fluorose moderada nos incisivos, caso que merece uma investigação clínica a fim de se pesquisar os possíveis fatores causais.

Admitindo-se, para o ano 2000, uma população residente de 2.280 crianças de 12 anos de idade na cidade (http://www.datasus.gov.br/ cgi/ibge/popmap.htm, acessado em 11/Jan/ 2001), pode-se estimar que cerca de 23 delas apresentam sinais em grau moderado. É provável que menos da metade delas esteja afetada por problemas estéticos, os quais dependem da intensidade e duração dos possíveis fatores causais, do período de exposição e a sua rela- ção com a cronologia de formação de cada grupo dentário de cada uma delas.

Taxas de fluorose dentária, entre 30,0-40,0\%, têm sido observadas em crianças do Estado da Paraíba, onde o teor de $0,7 \mathrm{ppm}$ de flúor é disponível 29. Em Porto Alegre, Rio Grande do Sul, a taxa de prevalência observada em adolescentes foi $52,9 \%$, e os pesquisadores não recomendaram qualquer medida de saúde pública 30 . Moysés et al. 14 avaliaram sua presença em escolares da rede pública da cidade de Curitiba, Paraná, e concluíram que a prevalência de $23,0 \%$ não constituía um problema epidemiológico relevante para a população escolar da cidade.

Em áreas (Iracemápolis e Piracicaba, Estado de São Paulo; Arroio do Tigre e Porto Alegre, Estado do Rio Grande do Sul) onde tem sido observada elevação da prevalência de fluorose na população infantil, a proporção de casos moderados/severos foi muito baixa 31,32 .

Clark \& Berkowitz 33 observaram que a prevalência de problemas estéticos decorrentes de fluorose dentária variou de 1 a $4 \%$ dependendo de como esses problemas eram definidos, mostrando que sua taxa pode ser baixa em comunidades com alta prevalência de fluorose.

Assim, cessar a fluoretação das águas de abastecimento sob a alegação de que essa medida é a causa do problema e sem a produção de conhecimentos mais consistentes pode significar prejuízo para a Saúde Coletiva. Estudos que avaliaram o que aconteceu quando houve descontinuidade dessa medida mostraram que o nível de cárie dentária se elevou 34,35,36,37. Para Narvai 38 (p. 386), “(...) considerando-se os conhecimentos disponíveis tais decisões não se justificam, sendo juridicamente ilegais, cientificamente insustentáveis e socialmente injustas".

Em locais com teores adequados de flúor na água de abastecimento, aspecto que implica em monitoramento periódico de sua concentração na ponta da rede, não se espera encontrar elevados percentuais de manifestações de fluorose incluídas nas condições moderada e severa, e, quando ocorre, predominam as formas leves, esteticamente aceitáveis. As taxas observadas entre os escolares do município superaram os padrões adotados por Dean, o que, na época, significava a desfluoretação das águas de abastecimento. Essa recomendação se justificava naquela situação em que a única fonte de exposição era a água de abastecimento "naturalmente" fluoretada e cujos teores eram superiores aos recomendados para prevenção da cárie dentária 39

A partir da segunda metade do século XX assistiu-se à expansão do uso do flúor na prevenção da cárie em larga escala, em todo o 
mundo, não apenas por meio de sua adição artificial nas águas de consumo, mas, também, através de outras formas como cremes dentais, medicamentos, polivitamíninos, colutórios, leite bovino e sal de cozinha, entre outras. Atualmente, a produção dos conhecimentos tem sido dirigida, entre outros aspectos, para a identificação dos fatores relacionados ao aumento da prevalência da fluorose nas populações. Investigações têm sido realizadas com o objetivo de avaliar os possíveis efeitos provocados pela redução do teor de flúor em cremes dentais e em águas de consumo a fim de controlar a exposição da comunidade a esse elemento químico sem prejudicar os resultados obtidos na prevenção da cárie dentária em crianças e adultos. O modo como o problema é percebido pela população também tem sido objeto de estudos a fim de contribuir para a determinação de padrões de aceitabilidade social.

Enquanto novos e mais conclusivos conhecimentos vêm sendo gerados, níveis muito baixos de fluorose esteticamente significativa decorrente de fontes múltiplas de flúor não justi-

\section{Resumo}

Fluorose dentária é uma opacidade do esmalte provocada pela ingestão de quantidades excessivas de flúor (exposição crônica) durante o período de formação dos dentes. A estimação desse problema em população exposta a fontes de flúor é necessária e deve ser feita periodicamente para monitorar os níveis e os padrões da alteração. A proposta desta pesquisa foi descrever e comparar dois estudos de prevalência para estimar a ocorrência de fluorose dentária na população do Município de Ribeirão Pires, Estado de São Paulo, Brasil, realizados em 1997 e 2000. Os métodos empregados em cada estudo foram brevemente apresentados. Os resultados foram comparados. Aspectos metodológicos e as implicações para Saúde Coletiva foram discutidos.

Odontologia em Saúde Coletiva; Fluorose Dentária; Estudos de Prevalência

\section{Colaboradores}

P. Frazão contribuiu na elaboração do artigo. A. C. Peverari e T. I. B. Forni produziram a primeira versão do manuscrito. Todas as seções do artigo foram revisadas e aprovadas por todos os autores. ficam a interrupção de medida tão ampla e eficaz no controle da cárie dentária. Assim, devese recomendar às autoridades sanitárias responsáveis por municípios semelhantes a Ribeirão Pires a manutenção das bases do sistema de prevenção adotado - água e creme dental fluorados - e maior precisão, rigor e ênfase nas medidas de vigilância sanitária 38,40 sobre esses e outros produtos fluorados, de modo a assegurar o máximo de benefício com o mínimo de risco de fluorose dentária.

Em relação à vigilância epidemiológica, o aspecto mais relevante que emerge da comparação dos resultados é a influência que a padronização de diferentes critérios pode ter exercido sobre as estimativas com e sem fluorose decorrente das categorias questionável e muito leve do instrumento de medida. Análises comparativas das estimativas de diferentes estudos devem ser cautelosas e admitir a possibilidade de considerar apenas as categorias de maior grau de severidade menos sujeitas às diferentes fontes de viés.

\section{Referências}

1. Fejerskov O, Thylstrup A, Larsen MJ. Clinical and structural features and possible pathogenic mechanisms of dental fluorosis. Scand J Dent Res 1977; 85:510-34.

2. Weatherell JA, Deutsch D, Robinson C, Hallsworth AS. Assimilation of fluoride by enamel throughout the life of the tooth. Caries Res 1977; 11 Suppl 1:85-115.

3. Den Besten PK. Biological mechanisms of dental fluorosis relevant to the use of fluoride supplements. Community Dent Oral Epidemiol 1999; 27:41-7.

4. Ripa LW. A critique of topical fluoride methods (dentifrices, mouthrinses, operator, and self-applied gels) in a era of decreased caries and increased fluorosis prevalence. J Public Health Dent 1991; 51:23-41.

5. Horowitz HS. The need for toothpaste with lower conventional fluoride concentrations for preschoolaged children. J Public Health Dent 1992; 52:216-21.

6. Lewis DW, Banting DW. Water fluoridation: current effectivenes and dental fluorosis. Community Dent Oral Epidemiol 1994; 22:153-8.

7. Kimmelman BB. Fluoride: is there a limit? Compend Contin Educ Dent 1995; 16:376-93.

8. Bawden JW. "Where is Waldo?": the timing of fluorosis. J Public Health Dent 1996; 56:5. 
9. Cangussu MCT, Narvai PC, Fernandez RC, Djehizian V. A fluorose dentária no Brasil: uma revisão crítica. Cad Saúde Pública 2002; 18:7-15.

10. Cury JA, Tabchoury CPM. Determination of appropriate exposure to fluoride in non-EME countries in the future. J Appl Oral Sci 2003; 11:83-95.

11. Forni TIB. Caracterização de levantamentos epidemiológicos de fluorose dentária no Estado de São Paulo [Dissertação de Mestrado]. São Paulo: Faculdade de Saúde Pública, Universidade de São Paulo; 2000.

12. World Health Organization. Oral health surveys: basic methods. $3^{\text {rd }}$ Ed. Geneva: World Health Organization; 1987.

13. World Health Organization. Oral health surveys: basic methods. $4^{\text {th }}$ Ed. Geneva: World Health Organization; 1997

14. Moysés SJ, Moysés ST, Allegretti ACV, Argenta M, Werneck R. Fluorose dental: ficção epidemiológica? Rev Panam Salud Publica 2002; 12:339-46.

15. Faculdade de Saúde Pública, Universidade de São Paulo/Secretaria de Estado de Saúde de São Paulo. Levantamento epidemiológico de cáries, oclusopatias e fluorose dentária, em escolares de 5 a 15 anos de idade, na região de saúde do $\mathrm{ABCD}, \mathrm{SP}$, Brasil, em 1997. Santo André: Faculdade de Saúde Pública, Universidade de São Paulo/Secretaria de Estado de Saúde de São Paulo; 2000. (Relatório técnico).

16. Peverari AC, Forni TIB, Frazão P. Prevalência de fluorose dentária em escolares no Município de Ribeirão Pires, Estado de São Paulo, Brasil, 2000. São Bernardo do Campo: Faculdade de Odontologia, Universidade Metodista de São Paulo; 2001. (Relatório de pesquisa).

17. Eklund SA, Moller IJ, Leclercq M. Calibration of examiners for oral health epidemiological surveys. Geneva: World Health Organization; 1993.

18. Dean HT, McKay FS. Production of mottled enamel halted by a change in the common water supply. In: McClure FJ, editor. Fluoride drinking waters. Bethesda: United States Public Health Service; 1939. p. 71-4.

19. Galagan DJ, Lamson GGJ. Climate and endemic dental fluorosis. In: McClure FJ, editor. Fluoride drinking waters. Maryland: United States Public Health Service; 1953. p.74-82.

20. Warnakulasuriya KA, Balasuriya S, Perera PAJ Peiris LC. Determining optimal levels of fluoride in drinking water for hot, dry climates - a case study in Sri Lanka. Community Dent Oral Epidemiol 1992; 20:364-7.

21. Rozier RG. Epidemiologic indices for measuring the clinical manifestations of dental fluorosis: overview and crititque. Adv Dent Res 1994; 8:39-55.

22. Kumar JV, Swango PA, Opima PN, Green EL. Dean's fluorosis index: an assessment of examiner reliability. J Public Health Dent 2000; 60:57-9.

23. Silva NN. Amostragem probabilística. São Paulo: Edusp; 1998.

24. Bragamian R. Workshop on "epidemiological indices of enamel defects”. Adv Dent Res 1989; 3:100.
25. Fejerskov O, Baelum V, Manji F, Moller IJ. Fluorose dentária: um manual para profissionais de saúde. São Paulo: Editora Santos; 1994.

26. Leverett D. Prevalence of dental fluorosis in fluoretaded and nonfluoretaded communities - preliminary investigation. J Public Health Dent 1986; 46:184-7.

27. Dean HT, Elvolve E. Further studies on the minimal threshold of chronic endemic dental fluorosis. In: McClure FJ, editor. Fluoride drinking waters. Bethesda: United States Public Health Service; 1937. p. 49-56.

28. Dean HT, Elvolve E, McKay FS. Mottled enamel survey of Bauxite, Ark., 10 years after a change in the common water supply. In: McClure FJ, editor. Fluoride drinking waters. Bethesda: United States Public Health Service; 1938. p. 56-62.

29. Sampaio FC. Prevalência de cárie e fluorose dentária em cidades da Paraíba com teores residuais de fluoretos na água de abastecimento. Rev Bras Ciênc Saúde 1993; 11:11-9.

30. Maltz M, Silva BB. Relação entre cárie, gengivite e fluorose e nível sócio-econômico em escolares. Rev Saúde Pública 2001; 35:170-6.

31. Maltz M, Silva BB, Schaeffer A, Farias C. Prevalência de fluorose em duas cidades brasileiras, uma com água artificialmente fluoretada e outra com baixo teor de flúor, em 1987 e 1997/98. Rev Fac Odontol P Alegre 2000; 41:51-5.

32. Pereira AC, Mialhe FL, Bianchini FLC, Meneghim MC. Prevalência de cárie e fluorose dentária em escolares de cidades com diferentes concentrações de flúor na água de abastecimento. Revista Brasileira de Odontologia em Saúde Coletiva 2001; 2 : 34-9.

33. Clark DC, Berkowitz J. The influence of various fluoride exposures on the prevalence of esthetic problems resulting from dental fluorosis. J Public Health Dent 1997; 57:144-9.

34. Lemke CW, Doherty JM, Arra MC. Controlled fluoridation: the dental effects of discontinuation in Antigo, Wisconsin. J Am Dent Assoc 1970; 80:782-6.

35. Kunzel W. Effect of an interruption in water fluoridation on the caries prevalence of the primary and secondary dentition. Caries Res 1980; 14:304-10.

36. Stephen KW, McCall DR, Tullis JI. Caries prevalence in northern Scotland before and 5 years after water defluoridation. Br Dent J 1987; 163:324-6.

37. Attwood D, Blinkhorn AS. Dental health of schoolchildren 5 years after water fluoridation ceased in southwest Scotland. Int Dent J 1991; 41:43-8.

38. Narvai PC. Cárie dentária e flúor: uma relação do século XX. Ciênc Saúde Coletiva 2000; 5:381-92.

39. Horowitz HS. Indexes for measuring dental fluorosis. J Public Health Dent 1986; 46:179-83.

40. Tomita NE, Panighel CPMA, Narvai PC, Lopes ES. Implicações da vigilância à saúde sobre ocorrência de fluorose dental. Rev ABO Nac 1995; 3:318-23.

Apresentado em 12/Nov/2003

Versão final reapresentada em 23/Mar/2004 Aprovado em 05/Abr/2004 